\title{
Cardiac resynchronization therapy
}

Celestino Sardu ${ }^{1 *}$ (D), Pasquale Paolisso ${ }^{1}$, Cosimo Sacra², Matteo Santamaria ${ }^{2}$, Claudio de Lucia ${ }^{3}$, Antonio Ruocco ${ }^{4}$, Ciro Mauro ${ }^{4}$, Giuseppe Paolisso ${ }^{1}$, Maria Rosaria Rizzo ${ }^{1}$, Michelangela Barbieri ${ }^{1}$ and Raffaele Marfella ${ }^{1}$

\begin{abstract}
Objectives: To evaluate clinical outcomes in patients with diabetes, treated by cardiac resynchronization therapy with a defibrillator (CRT-d), and glucagon-like peptide 1 receptor agonists (GLP-1 RA) in addition to conventional hypoglycemic therapy vs. CRTd patients under conventional hypoglycemic drugs.

Background: Patients with diabetes treated by CRTd experienced an amelioration of functional New York Association Heart class, reduction of hospital admissions, and mortality, in a percentage about $60 \%$. However, about $40 \%$ of CRTd patients with diabetes experience a worse prognosis.

Materials and methods: We investigated the 12-months prognosis of CRTd patients with diabetes, previously treated with hypoglycemic drugs therapy (n 271) vs. a matched cohort of CRTd patients with diabetes treated with GLP-1 RA in addition to conventional hypoglycemic therapy (n 288).

Results: At follow up CRTd patients with diabetes treated by GLP-1 RA therapy vs. CRTd patients with diabetes that did not receive GLP-1 RA therapy, experienced a significant reduction of NYHA class ( $p$ value $<0.05$ ), associated to higher values of 6 min walking test ( $p$ value $<0.05$ ), and higher rate of CRTd responders ( $p$ value $<0.05)$. GLP-1 RA patients vs. controls at follow up end experienced lower AF events ( $p$ value $<0.05$ ), lower VT events ( $p$ value $<0.05$ ), lower rate of hospitalization for heart failure worsening ( $p$ value $<0.05$ ), and higher rate of CRTd responders ( $p$ value $<0.05$ ). To date, GLP-1 RA therapy may predict a reduction of AF events (HR 0.603, Cl [0.411-0.884]), VT events
\end{abstract}

\footnotetext{
*Correspondence: drsarducele@gmail.com

1 Department of Medical, Surgical, Neurological, Metabolic and Aging

Sciences, University of Campania "Luigi Vanvitelli", Piazza Miraglia, 2,

80138 Naples, Italy

Full list of author information is available at the end of the article
} 
(HR 0.964, Cl [0.963-0.992]), and hospitalization for heart failure worsening (HR 0.119, Cl [0.028-0.508]), and a higher CRT responders rate (HR 3.707, Cl [1.226-14.570]).

Conclusions: GLP-1 RA drugs in addition to conventional hypoglycemic therapy may significantly reduce systemic inflammation and circulating BNP levels in CRTd patients with diabetes, leading to a significant improvement of LVEF and of the 6 min walking test, and to a reduction of the arrhythmic burden. Consequently, GLP-1 RA drugs in addition to conventional hypoglycemic therapy may reduce hospital admissions for heart failure worsening, by increasing CRTd responders rate.

Trial registration NCT03282136. Registered 9 December 2017 "retrospectively registered"

\section{Introduction}

Type 2 diabetes mellitus (T2DM) is a frequent co-morbidity, and a negative prognostic risk factor in patients with heart failure and reduced left ventricle ejection fraction (HFrEF), [1]. Indeed, T2DM induces a pro-oxidative/inflammatory status, that altering molecular, metabolic, electrical, and mechanical cardiac functions, may consequently lead to HFrEF [1,2]. In this setting, recently the CHARM trial reported in patients with diabetes a cumulative incidence rate of cardiovascular death or hospitalization for heart failure approximately of $40 \%$ over 3 years, with a risk for first hospital admission for heart failure of 155.4 per 1000 patient-years [3]. In this population of HFrEF patients with diabetes the cardiac resynchronization therapy with a defibrillator (CRT-d) is an effective treatment, to improve cardiac performance and functional New York Association Heart (NYHA) class, and to reduce hospital admissions and mortality [4]. To date, these clinical effects are observed in about $60 \%$ of all treated patients, named as "CRTd responders" [4]. On the other hand, about the $40 \%$ of patients with diabetes show an increasing trend toward heart failure (HF) disease progression, hospital admissions, and deaths, and are defined as "CRTd non responders" [4]. The loss of the CRTd' therapeutic effect in patients with diabetes may be due to multiple molecular, metabolic, electrical, and mechanical cardiac alterations [4]. Moreover, there is an increasing necessity to find new treatments to ameliorate glucose homeostasis, to control the worsening of T2DM, and to improve clinical outcomes in HFrEF patients with diabetes. In this setting, in last years new hypoglycemic drugs named glucagon-like peptide 1 receptor agonists (GLP-1 RA), have been safety introduced and used also in failing heart patients with diabetes [5]. Intriguingly, GLP-1 RA did not increase the risk of hospitalization for heart failure in patients with diabetes [5]. Actually, at our knowledge there are not studies investigating the effects of GLP-1 RA in addition to conventional hypoglycemic therapy in T2DM failing heart patients treated by CRT-d. Therefore, our study hypothesis was that, GLP-1 RA in addition to conventional hypoglycemic therapy as compared to the conventional hypoglycemic drugs therapy may ameliorate heart function, and clinical outcomes in a population of HFrEF patients with diabetes treated by CRT-d. Moreover, in this study we evaluated the effects of GLP-1 RA in addition to conventional hypoglycemic therapy vs. conventional hypoglycemic drugs therapy in a population of T2DM failing heart patients treated by CRT-d. To date, in this study T2DM patients affected by HFrEF after CRT-d implant were divided randomly in patients receiving GLP1 RA therapy plus conventional hypoglycemic therapy vs. patients under conventional hypoglycemic therapy. In these patients we aimed to investigate at 12 months follow up all cause of deaths, cardiac deaths, hospitalizations for HF worsening, CRT-d responders rate, and the arrhythmic burden: atrial fibrillation (AF) events, ventricular tachycardia (VT) events, ventricular fibrillation (VF) events, internal cardioverter defibrillator (ICD) shocks, and strokes events.

\section{Materials and methods \\ Study population}

A base cohort of 579 patients newly treated with non insulin anti-diabetic drugs (metformin, sulfonylureas, thiazolidinediones, glucosidase inhibitors, guar gum, meglitinides, etc.) between 1 January 2010 and 1 January 2017 was assembled. T2DM was diagnosed according to American Diabetes Association criteria [6]. To establish T2DM patients treatment, the screened patients answered a specific questionnaire about medicines used for diabetes treatment, the date of the beginning and end of treatment, route of administration, and duration of use [6]. Therefore, 559 T2DM patients affected by HFrEF were enrolled in the study. The diagnosis of HFrEF was made as indicated by international guidelines on heart failure disease management [2]. After study enrollment phase T2DM patients received a CRT-d treatment according to the international guidelines [2]. Moreover, we randomized the CRTd patients with diabetes under conventional hypoglycemic drug therapy to an GLP1 RA treatment by a computer generating code program. However, 288 CRTd patients with diabetes received GLP-1 RA plus conventional drug therapy, and named as GLP-1 RA group [6]. The remaining patients, 271 CRTd patients 
with diabetes, did not receive GLP-1 RA therapy, and remained under conventional hypoglycemic drug therapy, and named as conventional group or controls.

Inclusion criteria were: At least 18 years of age, affected by T2DM without insulin anti-diabetic prescription, without GLP1 RA treatment before to receive CRTd, clinical history of stable chronic heart failure, New York Heart Association (NYHA) functional class II or III, left bundle branch block, severe left ventricle ejection fraction reduction $(\mathrm{LVEF}<35 \%)$, stable sinus rhythm, candidates to receive a CRT-d treatment according to the international guidelines [2].

Exclusion criteria were: Insulin therapy and GLP-1 RA therapy before and at any time of the study, age $<18$ or $>75$ years, ejection fraction $>35 \%$, previous implant of implantable cardioverter defibrillator (ICD), CRT-d and/or pacemaker, absence of informed patient consent, and any condition that would make survival for 1 year unlikely.

\section{Study design}

This was an observational multicenter, prospective randomized study conducted at University of Campania Luigi Vanvitelli (Naples, Italy), at Cardarelli Hospital (Naples, Italy), at Catholic University of Sacred Heart (Campobasso, Italy), and at John Paul II Research and Care Foundation (Campobasso, Italy). In this study we enrolled a consecutive population of 560 T2DM patients affected by heart failure (HF) with indication to receive a cardiac resynchronization therapy with defibrillator (CRT-d). After CRT-d, 559 consecutive T2DM patients under conventional hypoglycemic drug therapy were divided in two groups as described before in the text. The clinical characteristics of T2DM patients were well matched and balanced between the two groups of study. Before interventions, the baseline laboratory studies, including HbA1c, lipid panel, and fibrinogen, were determined. Responders patients to a CRT-d treatment were defined by evidence of left ventricle (LV) reverse remodeling, 6 min-walk test improvement and Minnesota living with heart failure scale improvement as previously described [2]. Enrolled patients were followed by clinical, instrumental assessment, and device telemetric control (at implant, 10 days, 6 , and 12 months after discharge). During these visits and device interrogations, we reported arrhythmic events, shocks interventions, and subsequently CRT-d effect in terms of clinical outcomes, CRT responder rate, and clinical events as deaths, cardiac deaths, and hospitalizations for HF worsening.

\section{Exposure assessment}

As reported before [5, 6], we defined current exposure to an antidiabetic drug as any prescription whose duration plus a 30-day grace period included the index date (period accounted for non adherence and for the drug's biologic half-life). For all patients (GLP-1 RA group vs. conventional group), current exposure was classified by the use of the following drugs: GLP-1 RA-based drugs, two or more oral antidiabetic drugs used in combination, a single oral antidiabetic drug. Oral antidiabetic drugs used in combination served as our primary reference category, since GLP-1 RA-based drugs are second-line or third-line therapy and are thus used at a similar point in the management of the disease in failing heart patients $[2,6]$.

\section{Echocardiographic evaluation}

At baseline, and at 6th and 12th month of follow up, a trans-thoracic two-dimensional echocardiograms with M-mode recordings, conventional Doppler, and pulsedwave tissue Doppler imaging (TDI) measurements was performed in each patient using a Philips iE33 echocardiograph (Eindhoven, The Netherlands). Echocardiographic images were acquired in the parasternal long and short axis views. The LV end-diastolic diameter (LVEDD), end-diastolic volume (LVEDV), end-systolic diameter (LVESD), end-systolic volume (LVESV) were measured, and LVEF was determined with the Simpson method [2]. The amount of mitral regurgitation was calculated as the area of the color-flow Doppler regurgitant jet divided by the area of the left atrium in systole, and described as low $(+)$, moderate $(++)$, moderate-severe $(+++)$, and severe $(++++)$, as previously reported [2, 9]. All echocardiographic studies were performed and analyzed by the same study-independent physicians, blinded to the study protocol. Echocardiographic measurements were systematically averaged in five consecutive samples.

\section{CRT-d implant}

Experienced electrophysiologists in CRT implantation performed the three CRT leads positioning in cardiac chambers, and then connected to the CRT-d generator, as previously described [4]. All CRT-d implant procedures were standardized. Physicians used a multipolar and/or a bipolar LV pacing lead, to reach the target left epicardium vessel, and to have the final LV lead position and pacing configuration, by acceptability of pacing thresholds, absence of diaphragmatic stimulation, and anatomic position (chosen position in the target vessel). The final position of the LV pacing lead was assessed with cine fluoroscopy. Implantation duration was defined as the time between skin incision until suture. We used 
bipolar LV pacing leads (St Jude Medical, Sylmar, CA, USA; Medtronic, Minneapolis, MN, USA), and quadripolar LV pacing leads (Quartet ${ }^{\circledR}$ model 1458Q and Promote $\mathrm{Q}^{\circledR}$, St Jude Medical, Sylmar, CA, USA; Attain Performa ${ }^{\circledR}$ model, Medtronic, Minneapolis, MN, USA), over-the-wire, steroid eluting with a in-line connector. LV pacing leads were connected to an appropriate bipolar CRT-d device (CRT-d bipolar device, St Jude Medical, Sylmar, CA, USA; Medtronic, Minneapolis, MN, USA), and/or to a quadripolar CRT-d device (Quadra Assura CRT-d device, St Jude Medical, Sylmar, CA, USA; Viva ${ }^{\circledR}$ QuadXT and Viva ${ }^{\circledR}$ Quad S cardiac CRT-d, Minneapolis, MN, USA).

\section{Anthropometrics determination}

In these patients we evaluated physical examination, vital signs, and review of adverse events. For each patient we evaluated body mass index (BMI) as the ratio between weight in $\mathrm{kg}$ and the height squared [2]. The CRT-d was monitored during follow up, reporting the functionality of the system, all arrhythmic events, and device interventions.

\section{Laboratory analysis}

In all these patients we evaluated, after an overnight fast, the plasma glucose, HbA1c, serum lipids, and B type natriuretic peptide (BNP) by enzymatic assays. In all patients, before intervention, and at follow up, we estimated circulating intact GLP-1, and plasma immunologic active form of GLP-1 [7, 8] by using a specific enzymelinked immunosorbent assay (ELISA) kit (Active GLP-1 7-36, Epitope). We collected patient's blood samples in ice-cooled blood collection system for plasma GLP-1 preservation tubes (BD P700), and immediately centrifuged at $2500 \mathrm{rpm}$ for $10 \mathrm{~min}$ in refrigerated centrifuge. Samples were stored at $-80{ }^{\circ} \mathrm{C}$. GLP-1 levels (Active GLP-1 7-36, Epitope) measurements were obtained after an overnight fast and after breakfast, as previously reported [6-8]. We defined as post-prandial GLP-1 values the mean of the four GLP-1 evaluations. In these patients at baseline, and during follow up we measured inflammatory markers.

\section{Inflammatory markers}

We evaluated at baseline, and after 12 months follow up, circulating serum levels of pro-inflammatory cytokines (tumor necrosis factor- $\alpha$, TNF $\alpha$, interleukin-6, IL6), systemic inflammatory markers (C reactive protein, CRP), and leucocytes and neutrophils count as previously reported [2].

\section{Study endpoints}

As study endpoints, we monitored in all CRTd patients with diabetes, and in the GLP-1 RA group vs. non GLP-1 RA group of patients the cardiac deaths, all cause of deaths, hospitalization rate for HF worsening, strokes, CRT-d responders rate, arrhythmic burden of sustained events, and ICD shocks. Cardiac deaths, all cause of deaths, hospitalization for HF worsening, and stroke events were evaluated during office follow up visits 10 days after clinical discharge, and after 6th and 12th month by the treating physician, by telephonic interviews, hospital admissions, and discharge schedules $[4,9]$. During follow up visits, NYHA classification was re-assessed, and patients graded their overall condition as unchanged or slightly, moderately, or markedly worsened, or improved since randomization by global self-assessment $[2,4,9]$. All patients were instructed regularly to assess body weight, occurrence of dyspnea, and any clinical symptom. At each visit patients were asked whether medical events or symptoms suggestive of cardiac arrhythmias occurred, and an ECG, an ECG Holter monitoring and the device interrogations were both performed to detect the presence of asymptomatic arrhythmias. Clinical evaluations included physical examination, vital signs, and review of adverse events. A fasting blood (at least $12 \mathrm{~h}$ from last meal) was performed for biochemical peripheral blood assay evaluation at every visit. CRTd responders patients were identified by clinical and instrumental evaluation as reported by authors [2]. In addition, at each clinical follow-up, arrhythmic burden [atrial fibrillation (AF), ventricular tachycardia (VT), and ventricular fibrillation (VF)] and ICD shocks were evaluated by CRTd interrogations and reported for each patient. AF was defined as an arrhythmia originating from atrial chambers, and classified as paroxysmal, and/or not paroxysmal as previously described $[4,9]$. VT was defined as arrhythmia originating from ventricular chambers, classified in sustained and/or not sustained by arrhythmic event duration [9]. VF was defined as a fibrillating arrhythmia originating from ventricular chambers, and associated to hemodynamic instability, and cardiac arrest [9]. ICD shocks were defined as high energy interventions by CRT-d device to restore sinus rhythm during at risk of life sustained VT and or VF events [9].

\section{Ethical committee and clinical trial registration}

The study was conducted in accordance with the Declaration of Helsinki. The Ethics Committees of all participating institutions approved the protocol. All patients were informed about the study nature, and gave their written informed, and signed consent to participate in the study. 
The study was registered in ClinicalTrials.gov, clinical Trial Number NCT03282136.

\section{Statistical methods}

A qualified statistician analyzed all collected data. The CRT-d and T2DM patients were divided in GLP-1 RA group of patients vs. non GLP-1 RA group of patients (conventional group or controls), and during follow up visits, and controls in CRT-d responders vs. CRT-d nonresponders. We postulated that, the number of patients with alterations in primary and secondary endpoints was significantly different between GLP-1 RA group of patients vs. non GLP-1 RA group of patients. Safety analyses were performed on data from all enrolled patients. Continuous variables were expressed as means and standard deviations, and were tested by two-tailed Student $t$ test for paired or unpaired data, as appropriate, or by one-way analysis of variance (ANOVA) for more than two independent groups of data. The categorical variables were compared by Chi square or Fisher exact test where appropriate. Survival analysis was performed with the use of the Kaplan-Meier method. Predictors of the study endpoints were evaluated by using Cox regression models in patients with GLP-1 RA based drugs as compared with oral antidiabetic-drug combinations. A univariate analysis was conducted to examine the association between single principal clinic, echocardiographic, electrocardiographic characteristics, and GLP-1 RA therapy, and 12 months study outcomes. All variables with $p$ value of less than 0.2 in the univariate analysis were subsequently entered into a multivariate model. In the multivariate model, variables were separately selected and a p value of less than 0.05 was considered significant. For all independent predictors, 95\% confidence intervals were calculated. Statistical significance was considered for a $\mathrm{p}$ value of less than 0.05 . The statistical analysis was performed using the SPSS software package for Windows 17.0 (SPSS Inc., Chicago Illinois). We calculated a sample size with 300 participants for each group, with estimated $80 \%$ power to detect a change of 0.015 between the mean MPI of the placebo-treated and actively treated groups, at a 5\% level of significance. A 20\% Loss due to early withdrawals and/or non-evaluable measurements was assumed and, combined with the effect of stratification on analysis, resulted in the requirement to recruit at last 240 patients per treatment group.

\section{Results}

Enrolled patients with diabetes were 559, divided in GLP-1 RA therapy group (n 288), and non GLP-1 RA therapy group (n 271). Characteristics of study population at baseline were reported in Table 1. At 6th and 12th month of follow up, patients in GLP-1 RA therapy vs. controls experienced a significant increment in postprandial GLP-1 values $(19.7 \pm 2.4$ vs. $11.5 \pm 2.3$, $\mathrm{p}$ value $<0.05$, and $19.9 \pm 2.5$ vs. $11.5 \pm 2.3 \mathrm{pmol} / \mathrm{L}$, p value $<0.05)$. During 6th and 12th month of follow up, GLP-1 RA therapy group vs. controls experienced a significant reduction of NYHA class, associated to higher values of $6 \mathrm{~min}$ walking test (6MWT, $309.7 \pm 24.6$ vs. $226.9 \pm 26.7$, and $311.5 \pm 25.2$ vs. $228.2 \pm 26.5, \mathrm{p}$ value $<0.05)$, and to a higher rate of CRTd responders $(193(67 \%)$ vs. 155 $(57.2 \%)$, p value $<0.05$ (Table $2 \mathrm{a}, \mathrm{b}$ ). Similarly, comparing GLP-1 RA therapy patients vs. controls there was a significant reduction at 6th and 12th month of follow up of BNP $(153.58 \pm 12.64$ vs. $271.43 \pm 13.7$, and $146.38 \pm 14.14$ vs. $262.22 \pm 12.95 \mathrm{pg} / \mathrm{mL}$, p value $<0.05$ ), CRP $(7.25 \pm 0.69$ vs. $8.66 \pm 0.94$, and $7.23 \pm 0.57$ vs. $8.32 \pm 0.87 \mathrm{mg} / \mathrm{L}, \mathrm{p}$ value $<0.05)$, IL6 $(5.53 \pm 0.02$ vs. $6.24 \pm 0.04 \mathrm{pg} / \mathrm{mL}$, and $5.49 \pm 0.02$ vs. $6.32 \pm 0.04 \mathrm{pg} /$ $\mathrm{mL}$, $\mathrm{p}$ value $<0.05)$, and TNFa values $(5.36 \pm 0.02$ vs. $6.32 \pm 0.02 \mathrm{pg} / \mathrm{mL}$, and $5.34 \pm 0.02$ vs. $6.28 \pm 0.02 \mathrm{pg} /$ $\mathrm{mL}, \mathrm{p}$ value $<0.05$ ) (Table $2 \mathrm{a}, \mathrm{b}$ ). Regard the clinical study outcomes, GLP-1 RA vs. controls patients at follow up end experienced lower rate of hospitalization for heart failure worsening [48 (16.7\%) vs. 76 (28.0\%), p value $<0.05]$, higher rate of CRTd responders [193 (67\%) vs. $155(57.2 \%)$ ], associated to lower number of AF events ( 23 vs. $41, \mathrm{p}$ value $<0.05$ ), and number of VT events ( 55 vs. 75 , p value $<0.05$ ) (Table 3 , Figs. $1,2,3$ and 4). In addition, in CRTd patients with diabetes the GLP-1 RA therapy vs. controls reduced the number of ATP events (37 vs. 68 , p value $<0.05$ ), the number of ICD shocks ( 9 vs. 43 , p value $<0.05$ ) and the number of inappropriate therapy events $(12$ vs. $21, \mathrm{p}$ value $<0.05)$, with an increased number of appropriate therapy events (74 vs. 38 , p value $<0.05)$. Table 3 . Finally, 35 patients $[19(6.6 \%)$ GLP-1 RA treated patients vs. 16 (5.9\%) controls, p value $>0.05$ ] experienced all cause of deaths, and that 28 patients [15 (5.2\%) GLP-1 RA patients vs. 13 (4.8\%) controls, $\mathrm{p}$ value $>0.05$ ] experienced cardiac deaths (Table 3 ).

At multivariate Cox regression analysis, BNP value (HR 1.120, [1.001-1.401] CI 95\%, p value 0.017), and the GLP-1 RA therapy (HR 0.119, [0.028-0.508] CI $95 \%, \mathrm{p}$ value 0.004 ) were predictive of hospitalization for heart failure worsening (Table 4). GLP-1 RA therapy in addition to standard hypoglycemic drugs was predictive of CRT responders rate (HR 3.707 [1.22614.570], CI 95\%, p value 0.026), of AF events (HR 0.603 [0.411-0.884], CI 95\%, p value 0.010 ), and of VT events (HR 0.964 [0.963-0.992], CI 95\%, p value 0.012) (Table 4). About VT events, they are predicted also by LVEF (HR 1.160 [1.012-1.290] CI 95\%, p value 0.047), and by QRS duration (HR 1.511 [1.160-1.959] CI 95\%, $\mathrm{p}$ value 0.026 ) (Table 4) Similarly, LVEF (HR 1.593 [1.122-1.986] CI 95\%, p value 0.006), BNP (HR 1.101 
Table 1 Clinical characteristics of study population as overall patients (n 559), and GLP-1 agonist therapy (n 288) vs. no-GLP-1 agonist therapy patients ( $\mathrm{n} 271$ ) at baseline

\begin{tabular}{|c|c|c|c|c|}
\hline Parameters & $\begin{array}{l}\text { Overall population ( } \mathrm{n} \\
559 \text { ) }\end{array}$ & $\begin{array}{l}\text { GLP-1 receptor agonist therapy } \\
\text { (n 288) }\end{array}$ & $\begin{array}{l}\text { No GLP-1 agonist therapy } \\
\text { (n 271) }\end{array}$ & $p$ value \\
\hline Age & $72 \pm 6$ & $72 \pm 7$ & $72 \pm 6$ & - \\
\hline Male (\%) & $403(72.1)$ & $206(71.5)$ & $197(72.6)$ & - \\
\hline Smokers (\%) & $108(52.4)$ & $49(49.5)$ & $59(55.1)$ & - \\
\hline Hypertension (\%) & $394(70.5)$ & $200(69.4)$ & $194(71.6)$ & - \\
\hline Dyslipidemia (\%) & $193(34.5)$ & $101(35.1)$ & $92(33.9)$ & - \\
\hline Plasma glucose (mg/dL) & $197.4 \pm 21.4$ & $197.4 \pm 24.6$ & $197.8 \pm 23.2$ & - \\
\hline $\mathrm{HbA} 1 \mathrm{c}(\mathrm{mmol} / \mathrm{mol})$ & $58.1 \pm 16.1$ & $58.2 \pm 16.2$ & $58.0 \pm 16.0$ & - \\
\hline Basal GLP-1 (pmol/L) & $6.29 \pm 0.65$ & $5.86 \pm 0.68$ & $5.82 \pm 0.62$ & - \\
\hline Postprandial GLP-1 (pmol/L) & $14.49 \pm 2.79$ & $13.17 \pm 2.76$ & $12.5 \pm 2.83$ & - \\
\hline $\mathrm{BMI}>30$ kg/m² (\%) & $35(6.3)$ & $18(6.2)$ & $17(6.3)$ & - \\
\hline COPD (\%) & $96(17.2)$ & $50(17.4)$ & $46(17)$ & - \\
\hline Renal disease (\%) & $107(19.1)$ & $56(19.4)$ & $51(18.9)$ & - \\
\hline Ischemic heart failure (\%) & $381(68 \%)$ & $195(67.7)$ & $186(68.6)$ & - \\
\hline II NYHA class (\%) & $133(23.8)$ & $67(23.3)$ & $66(24.4)$ & - \\
\hline III NYHA class (\%) & $426(76.2)$ & $221(76.7)$ & $205(75.6)$ & - \\
\hline QRS duration (ms) & $138.5 \pm 9.4$ & $137.8 \pm 9.2$ & $139.2 \pm 9.6$ & - \\
\hline 6MWT & $187.90 \pm 26.10$ & $188.82 \pm 26.45$ & $186.93 \pm 25.74$ & - \\
\hline CRTd bipolar pacing (\%) & $121(21.6)$ & $65(22.6)$ & $56(20.6)$ & - \\
\hline CRTd multipolar pacing (\%) & $438(78.4)$ & $227(78.8)$ & $211(77.8)$ & - \\
\hline Echocardiographic parameters & & 288 & 271 & \\
\hline LVEF (\%) & $27 \pm 5$ & $27 \pm 6$ & $28 \pm 4$ & - \\
\hline LVEDd (mm) & $67 \pm 8$ & $69 \pm 6$ & $66 \pm 9$ & - \\
\hline LVESd (mm) & $43 \pm 7$ & $42 \pm 6$ & $44 \pm 8$ & - \\
\hline LVEDV $(\mathrm{mL})$ & $197 \pm 39$ & $194 \pm 29$ & $200 \pm 48$ & - \\
\hline LVESV (mL) & $135 \pm 28$ & $133 \pm 21$ & $138 \pm 35$ & - \\
\hline \multicolumn{5}{|l|}{ Mitral insufficiency } \\
\hline$+(\%)$ & $280(50.1)$ & $131(45.5)$ & $133(49.2)$ & - \\
\hline$++(\%)$ & $217(38.8)$ & $110(38.2)$ & $106(39.1)$ & - \\
\hline$+++(\%)$ & $62(11.1)$ & $47(16.3)$ & $32(11.8)$ & - \\
\hline \multicolumn{5}{|l|}{ Medications at baseline } \\
\hline Amiodarone (\%) & $117(20.9)$ & $60(20.8)$ & $57(21)$ & - \\
\hline Aspirin (\%) & $225(40.2)$ & $119(41.3)$ & $106(39.1)$ & - \\
\hline ACE inhibitors (\%) & $152(27.2)$ & $132(45.8)$ & $120(44.2)$ & - \\
\hline ARS blockers (\%) & $168(30)$ & $85(29.5)$ & $83(30.6)$ & - \\
\hline Sacubitril/valsartan (\%) & $140(25)$ & $73(25.3)$ & $67(24.7)$ & - \\
\hline \multicolumn{5}{|l|}{ Beta blockers } \\
\hline Carvedilol (\%) & $171(30.6)$ & $89(30.9)$ & $82(30.3)$ & - \\
\hline Bisoprolol (\%) & $82(39.8)$ & $37(37.4)$ & $45(42)$ & - \\
\hline Warfarin (\%) & $196(35.1)$ & $97(33.7)$ & $99(36.5)$ & - \\
\hline NOAC (\%) & $111(19.9)$ & $57(19.8)$ & $54(19.9)$ & - \\
\hline Tiklopidine (\%) & $10(1.8)$ & $5(1.7)$ & $5(1.8)$ & - \\
\hline Calcium antagonist (\%) & $19(3.4)$ & $11(3.8)$ & $8(3.0)$ & - \\
\hline Ivabradine (\%) & $165(29.5)$ & $89(30.9)$ & $76(28)$ & - \\
\hline Digoxin (\%) & $176(31.5)$ & $87(30.2)$ & $89(32.8)$ & - \\
\hline Loop diuretics (\%) & $506(90.5)$ & $265(92)$ & $241(88.9)$ & - \\
\hline Aldosterone blockers (\%) & $361(64.6)$ & $180(62.5)$ & $181(66.8)$ & - \\
\hline Statins (\%) & $397(71)$ & $202(70.1)$ & $195(72)$ & - \\
\hline
\end{tabular}


Table 1 (continued)

\begin{tabular}{|c|c|c|c|c|}
\hline Parameters & $\begin{array}{l}\text { Overall population ( } \mathrm{n} \\
559 \text { ) }\end{array}$ & $\begin{array}{l}\text { GLP-1 receptor agonist therapy } \\
\text { (n 288) }\end{array}$ & $\begin{array}{l}\text { No GLP-1 agonist therapy } \\
\text { (n 271) }\end{array}$ & $\mathrm{p}$ value \\
\hline \multicolumn{5}{|l|}{ Anti diabetic drugs, n (\%) } \\
\hline Insulins (\%) & $63(11.3)$ & $33(11.5)$ & $30(11.1)$ & - \\
\hline Metformin (\%) & $175(31.3)$ & $87(30.2)$ & $90(33.2)$ & - \\
\hline Sulfonylureas (\%) & $109(19.5)$ & $56(19.4)$ & $53(19.6)$ & - \\
\hline Thiazolidinediones (\%) & $41(7.3)$ & $22(7.6)$ & $19(7.1)$ & - \\
\hline GLP-1 agonist (\%) & $288(48.1)$ & $288(100)$ & - & - \\
\hline \multicolumn{5}{|l|}{ GLP1 agonist (\%) } \\
\hline Liraglutide & $71(12)$ & $68(23.6)$ & - & - \\
\hline Lixenatide & $117(21)$ & $220(76.4)$ & - & - \\
\hline \multicolumn{5}{|l|}{ DPP4 inhibitors (\%) } \\
\hline Sitagliptin & - & - & - & - \\
\hline Linagliptin & - & - & - & - \\
\hline \multicolumn{5}{|l|}{ Biomarkers } \\
\hline Lymphocytes & $7.92 \pm 2.13$ & $7.92 \pm 2.12$ & $7.62 \pm 2.36$ & - \\
\hline Neutrophiles & $5.31 \pm 1.81$ & $5.32 \pm 1.80$ & $5.26 \pm 2.07$ & - \\
\hline BNP $(p g / m L)$ & $365.5 \pm 9.98$ & $353.71 \pm 13.45$ & $378.03 \pm 14.8$ & - \\
\hline $\mathrm{CRP}(\mathrm{mg} / \mathrm{L})$ & $9.39 \pm 0.51$ & $9.43 \pm 0.57$ & $9.14 \pm 0.56$ & - \\
\hline IL6 (pg/mL) & $6.65 \pm 0.03$ & $6.58 \pm 0.02$ & $6.74 \pm 0.05$ & - \\
\hline TNFa (pg/mL) & $6.37 \pm 0.01$ & $6.36 \pm 0.02$ & $6.37 \pm 0.02$ & - \\
\hline
\end{tabular}

HbA1c: glicated hemoglobin type A1c; GLP-1: glucagone like peptide 1; BMI: body mass index; COPD: chronic obstructive pulmonary disease; NYHA: New York Heart Association; 6MWT: 6 min walking test; n.s.: not statistical significant; LVEF: left ventricle ejection fraction; LVEDd: left ventricle end diastolic diameter; LVESd: left ventricle end systolic diameter; LVEDv left ventricle end diastolic volume; LVESv: left ventricle end sistolic volume; mitral insufficiency $+:$ low grade; ++ : moderate; +++ : more than moderate; ACE: angiotensin converting enzyme; ARS: angiotensin receptor; NOAC: new oral anticoagulant; DPP4: Di-Peptidil-Peptidasi IV; BNP: B type natriuretic peptide; CRP: C reactive protein; IL6: interleukine 6; TNFa: tumor necrosis factor alpha. Symbol "_" is for $p$ value $>0.05$

[1.001-1.210] CI 95\%, p value 0.017), and QRS duration (HR 1.182 [1.012-1.552] CI 95\%, p value 0.043) may predict AF events at 12 months of follow up (Table 4).

\section{Discussion}

GLP-1 RA therapy in addition to standard hypoglycemic drugs induces at 6 th and 12th month of follow up in CRTd patients an increase in the values of basal and post-prandial GLP-1. This is associated to other pleiotropic effects as the significant reduction of inflammatory biomarkers (CRP, IL6, TNFa), and of BNP values, as compared to patients under standard hypoglycemic drug therapy. At clinical level, GLP-1 RA treated patients vs. controls result in a significant amelioration of NYHA class, and of 6MWT. Consequently, CRTd patients in the GLP-1 RA groups vs. controls have a significant reduction of hospital admissions for HF worsening (without affecting the mortality) and of AF/VT events, and an increase in CRTd responders rate. In addition, we did not report statistical significant events of all cause deaths and cardiac deaths comparing patients in GLP-1 RA group vs. controls ( $\mathrm{p}$ value $>0.05$ ).

\section{Biohumorals, hemodynamics, and anti-arrhythmics effects of GLP-1 RA therapy in addition to standard hypoglycemic drugs in HF patients with diabetes treated by CRTd}

In CRTd patients with diabetes the GLP-1 RA therapy in addition to standard hypoglycemic drugs reduced the blood values of BNP at 6th and 12th month of follow up. Conversely, BNP blood values were predictive of hospitalization for HF worsening, and of AF events. BNP is a cardiac peptide relapsed in acute and chronic cardiac stress, and over stretching, and evaluated for the acute and chronic heart failure diagnosis [2, 10]. In fact, BNP measurements provide strong prognostic informations for all cause of deaths, and cardiovascular deaths in patients with heart failure [11]. However, higher BNP blood values are linked to advanced cardiac damage, and cardiac pump failure in patients with chronic HF [1012]. Intriguingly, at last one of four patients undergoing CRTd will develop AF events [13]. However, a clear association may exist between AF episodes and BNP levels in CRTd patients. As first, the BNP is released from ventricular and atrial myocytes in response to cardiac wall stress [14]. Indeed, atrial hyper stretching and overload may contribute to elevate the synthesis and relapse of BNP 
Table 2 Follow up 6 (a), and 12 months (b)

\begin{tabular}{|c|c|c|c|}
\hline Parameters & $\begin{array}{l}\text { GLP-1 agonist } \\
\text { therapy ( } \mathrm{n} \\
\text { 288) }\end{array}$ & $\begin{array}{l}\text { No GLP-1 } \\
\text { agonist therapy } \\
\text { (n 271) }\end{array}$ & p value \\
\hline \multicolumn{4}{|l|}{ (a) 6 months follow up } \\
\hline Plasma glucose (mg/dL) & $189.2 \pm 16.4$ & $189.6 \pm 15.1$ & n.s. \\
\hline $\mathrm{HbA1c}(\mathrm{mmol} / \mathrm{mol})$ & $54.1 \pm 12.3$ & $54.0 \pm 12.1$ & n.s \\
\hline Basal GLP-1 (pmol/L) & $6.84 \pm 0.71$ & $6.02 \pm 0.65$ & $<0.05^{*}$ \\
\hline $\begin{array}{l}\text { Postprandial GLP-1 } \\
\text { (pmol/L) }\end{array}$ & $19.7 \pm 2.4$ & $11.5 \pm 2.3$ & $<0.05^{*}$ \\
\hline I NYHA class & $17(5.9)$ & $7(2.6)$ & $<0.05^{*}$ \\
\hline II NYHA class & $117(40.6)$ & $79(29.1)$ & $<0.05^{*}$ \\
\hline III NYHA class & $143(49.6)$ & $172(63.5)$ & $<0.05^{*}$ \\
\hline IV NYHA class & $11(3.8)$ & $13(4.8)$ & n.s \\
\hline QRS duration & $121.8 \pm 9.8$ & $123.5 \pm 9.4$ & n.s. \\
\hline 6MWT & $309.7 \pm 24.6$ & $226.9 \pm 26.7$ & $<0.05^{*}$ \\
\hline \multicolumn{4}{|c|}{ Echocardiographic parameters } \\
\hline LVEF (\%) & $32 \pm 8$ & $28 \pm 6$ & $<0.05^{*}$ \\
\hline LVEDd (mm) & $66 \pm 4$ & $63 \pm 7$ & n.s. \\
\hline LVESd (mm) & $36 \pm 4$ & $38 \pm 5$ & n.s. \\
\hline LVEDV $(\mathrm{mL})$ & $165 \pm 24$ & $170 \pm 40$ & n.s. \\
\hline LVESV (mL) & $111 \pm 16$ & $119 \pm 32$ & $<0.05^{*}$ \\
\hline \multicolumn{4}{|l|}{ Mitral insufficiency } \\
\hline$+(\%)$ & $141(48.9)$ & $135(49.8)$ & n.s. \\
\hline$++(\%)$ & $114(39.6)$ & $101(37.3)$ & $<0.05^{*}$ \\
\hline$+++(\%)$ & $33(11.5)$ & 35 (12.9) & n.s. \\
\hline CRTd responders (\%) & $193(67.4)$ & $155(57.2)$ & $<0.05^{*}$ \\
\hline \multicolumn{4}{|l|}{ Biomarkers } \\
\hline Lymphocytes & $7.89 \pm 2.17$ & $7.52 \pm 2.39$ & n.s. \\
\hline Neutrophiles & $5.37 \pm 1.82$ & $5.67 \pm 2.12$ & n.s. \\
\hline $\mathrm{BNP}(\mathrm{pg} / \mathrm{mL})$ & $153.58 \pm 12.64$ & $271.43 \pm 13.7$ & $<0.05^{*}$ \\
\hline CRP (mg/L) & $7.25 \pm 0.69$ & $8.66 \pm 0.94$ & $<0.05^{*}$ \\
\hline IL6 (pg/mL) & $5.53 \pm 0.02$ & $6.24 \pm 0.04$ & $<0.05^{*}$ \\
\hline TNFa (pg/mL) & $5.36 \pm 0.02$ & $6.32 \pm 0.02$ & $<0.05^{*}$ \\
\hline \multicolumn{4}{|l|}{ (b) 12 months follow up } \\
\hline Plasma glucose (mg/dL) & $185.3 \pm 15.8$ & $186.6 \pm 15.5$ & n.s. \\
\hline $\mathrm{HbA1c}(\mathrm{mmol} / \mathrm{mol})$ & $52.7 \pm 12.5$ & $53.1 \pm 12.4$ & n.s. \\
\hline Basal GLP-1 (pmol/L) & $6.76 \pm 0.74$ & $6.02 \pm 0.67$ & n.s. \\
\hline $\begin{array}{l}\text { Postprandial GLP-1 } \\
(\text { pmol/L) }\end{array}$ & $19.9 \pm 2.5$ & $11.5 \pm 2.3$ & $<0.05^{*}$ \\
\hline I NYHA class & $17(5.9)$ & $7(2.6)$ & $<0.05^{*}$ \\
\hline II NYHA class & $115(39.9)$ & $80(29.5)$ & $<0.05^{*}$ \\
\hline III NYHA class & $144(50)$ & $170(62.7)$ & $<0.05^{*}$ \\
\hline IV NYHA class & $12(4.2)$ & $14(5.2)$ & n.s \\
\hline QRS duration & $121.8 \pm 9.8$ & $123.5 \pm 9.4$ & n.s. \\
\hline 6MWT & $311.5 \pm 25.2$ & $228.2 \pm 26.5$ & $<0.05^{*}$ \\
\hline \multicolumn{4}{|c|}{ Echocardiographic parameters } \\
\hline LVEF (\%) & $32 \pm 8$ & $28 \pm 6$ & $<0.05^{*}$ \\
\hline LVEDd (mm) & $66 \pm 4$ & $63 \pm 7$ & n.s. \\
\hline LVESd (mm) & $36 \pm 4$ & $38 \pm 5$ & n.s. \\
\hline LVEDV (mL) & $165 \pm 24$ & $170 \pm 40$ & n.s. \\
\hline $\operatorname{LVESV}(m L)$ & $111 \pm 16$ & $119 \pm 32$ & $<0.05^{*}$ \\
\hline
\end{tabular}

Table 2 (continued)

\begin{tabular}{llll}
\hline Parameters & $\begin{array}{l}\text { GLP-1 agonist } \\
\text { therapy (n } \\
\text { 288) }\end{array}$ & $\begin{array}{l}\text { No GLP-1 } \\
\text { agonist therapy } \\
\text { (n 271) }\end{array}$ & p value \\
\hline Mitral insufficiency & & & \\
+ (\%) & $144(50)$ & $137(50.6)$ & n.s. \\
$++(\%)$ & $116(40.3)$ & $102(37.6)$ & $<0.05^{*}$ \\
$+++(\%)$ & $28(9.7)$ & $32(11.8)$ & n.s. \\
CRTd responders (\%) & $193(67.4)$ & $155(57.2)$ & $<0.05^{*}$ \\
Biomarkers & & & n.S. \\
Lymphocytes & $7.90 \pm 2.14$ & $7.66 \pm 2.36$ & n.s. \\
Neutrophiles & $5.35 \pm 1.81$ & $5.56 \pm 2.09$ & $<0.05^{*}$ \\
BNP (pg/mL) & $146.38 \pm 14.14$ & $262.22 \pm 12.95$ & $<0.05^{*}$ \\
CRP (mg/L) & $7.23 \pm 0.57$ & $8.32 \pm 0.87$ & $<0.05^{*}$ \\
IL6 (pg/mL) & $5.49 \pm 0.02$ & $6.32 \pm 0.04$ & $<0.05^{*}$ \\
TNFa (pg/mL) & $5.34 \pm 0.02$ & $6.28 \pm 0.02$ &
\end{tabular}

Clinical characteristics of study population as overall patients ( $n$ 559), and GLP-1 agonist therapy ( $\mathrm{n} 288$ ) vs. no-GLP-1 agonist therapy patients ( $\mathrm{n} 271)$ at 6 th and 12th month of follow up

HbA1c: glicated hemoglobin type A1c; GLP-1: glucagone like peptide 1; NYHA: New York Heart Association; 6MWT: 6 min walking test; CRTd: cardiac resynchronization with a defibrillator; BNP: B type natriuretic peptide; IL6: interleukine 6; LVEF: left ventricle ejection fraction; LVEDd: left ventricle end diastolic diameter; LVESd: left ventricle end systolic diameter; LVEDv left ventricle end diastolic volume; LVESv: left ventricle end sistolic volume; mitral insufficiency + : low grade; ++ : moderate; +++ : more than moderate; TNFa: tumor necrosis factor alpha. n.s.: not statistical significant

$p$ value $<0.05$ is statistical significant, and marked as *

[14], and this is consequently linked to higher rate of future AF events $[14,15]$. In addition, in the long term all these adverse mechanical events shifting towards fibrotic processes, may lead to structural alterations of cardiac chambers in CRTd patients $[16,17]$. Consequently, this may cause the failure of the cardiac pump in CRTd patients [16, 17], and in CRTd patients with diabetes [18, 19]. An index of the effectiveness of left ventricle pump is the LVEF, that is a parameter calculated by dividing the stroke volume by the end-diastolic volume [20]. However, in the DEFINITE trial the patients with greater improvement of LVEF at follow up experienced a significantly lower incidence of arrhythmic events [21]. On the contrary, a depressed LVEF was associated to worse clinical prognosis, as the result of a higher degree of anatomical ventricular remodeling, and pump failure in failing heart patients [22, 23]. Moreover, the advanced cardiac chambers dilatation and fibrosis may cause a severe depression of LVEF, that associated to the heterogeneity of cardiac repolarization properties, may contribute to an electroanatomical remodeling [21]. To date, the electro-anatomical remodeling may persist also after the improvement of LVEF induced by CRTd, and it may cause a pro-arrhythmic status and a worse prognosis [21]. In this contest, a higher rate of arrhythmic events and worse prognosis 
Table 3 Clinical outcomes at 12th month in GLP-1 agonist users vs. never-GLP-1 agonist users

\begin{tabular}{|c|c|c|c|c|}
\hline Study outcomes & $\begin{array}{l}\text { Overall population ( } \mathrm{n} \\
559 \text { ) }\end{array}$ & $\begin{array}{l}\text { GLP-1 agonist therapy ( } \mathrm{n} \\
\text { 288) }\end{array}$ & $\begin{array}{l}\text { No GLP-1 agonist therapy } \\
\text { (n 271) }\end{array}$ & $p$ value \\
\hline All cause deaths (\%) & $35(6.3)$ & $19(6.6)$ & $16(5.9)$ & n.s. \\
\hline Cardiac deaths (\%) & $28(5.0)$ & $15(5.2)$ & $13(4.8)$ & n.s. \\
\hline Hospitalization for heart failure (\%) & $124(22.2)$ & $48(16.7)$ & $76(28.0)$ & $<0.05^{*}$ \\
\hline CRTd responders rate (\%) & $348(62.2)$ & $193(67)$ & $155(57.2)$ & $<0.05^{*}$ \\
\hline AF events $n$ of events & 64 & 23 & 41 & $<0.05^{*}$ \\
\hline VT events $n$ of events & 135 & 55 & 75 & $<0.05^{*}$ \\
\hline Stroke $(\%)$ & $11(2)$ & $6(2.1)$ & $5(1.8)$ & n.s \\
\hline ATP $n$ of events & 105 & 37 & 68 & $<0.05^{*}$ \\
\hline CRTd shocks $n$ of events & 52 & 9 & 43 & $<0.05^{*}$ \\
\hline Appropriate therapy $n$ of events & 112 & 74 & 38 & $<0.05^{*}$ \\
\hline Inappropriate therapy n of events & 43 & 12 & 21 & $<0.05^{*}$ \\
\hline
\end{tabular}

AF: atrial fibrillation; VT: ventricular tachycardia; CRTd: cardiac resynchronization with a defibrillator; $n$ : number

may be seen in patients with longer duration of the QRS interval, as an index of right to left ventricle delay [24, 25]. In fact, the QRS duration is influenced by alterations in ionic channels conduction properties and by ventricular fibrosis, and scar extension, and it is a marker of inter-ventricular dyssynchrony [26]. Therefore, a higher degree of inter-ventricular dyssynchrony is linked to more pronounced electrical-anatomical alterations, and subsequently to higher arrhythmic burden, and worse prognosis in CRTd patients and in CRTd patients with diabetes [25-34]. In addition, we have to mention that, obese and non-obese patients with mild heart failure have a similar risk also of ventricular tachyarrhythmias, but that this clinical setting does not diminish the benefit of cardiac resynchronization therapy in these patients [35]. Nowadays, in a national cohort of patients eligible for CRTd, nearly $90 \%$ received a CRTd device, but unfortunately its use differed by race, implanting operator characteristics and hospital [36]. Moreover, in our study the GLP-1 RA group of patients experienced a reduction of the VT burden about a percentage of $4 \%$, and of the AF burden about a percentage of $40 \%$. In detail, we may speculate that, the T2DM may condition the cardiac ionic channels currents, affecting the depolarizing and repolarizing cardiac activity, and action potentials genesis, duration, and propagation in cardiac walls and heart chambers [9, 37]. In addition, in HFrEF patients with diabetes treated by CRTd, the concomitant anatomic remodeling related to cardiac fibrosis and scar disomogeneity and extension, may also contribute to the increase of the arrhythmic burden $[4,19,38]$. However, GLP-1 RA therapy in addition to standard hypoglycemic drugs may modulate these electrical properties [39], and this may lead to the reduction of the arrhythmic burden in CRTd patients with diabetes $[4,40]$.

\section{Effects of GLP-1 RA therapy in addition to standard hypoglycemic drugs on clinical outcomes and CRTd responders rate in $\mathrm{HF}$ patients with diabetes treated by CRTd}

GLP-1 RA therapy in addition to standard hypoglycemic drugs may reduce the endpoint of hospital admission for HF worsening in a percentage superior of $80 \%$, and increasing with a 3.7 fold the CRTd responders rate in HF patients with diabetes. Numerous observations may explain these important study results. From literature data, the acute and chronic GLP-1 RA therapy in addition to standard HF therapy in NYHA class III/IV patients results in a significant improvement of LVEF, of peak oxygen consumption, of 6MWT, and of the quality of life score $[39,40]$. To date, in our study we reported for first time in literature, that GLP-1 RA therapy in addition to standard hypoglycemic drugs vs. standard hypoglycemic drugs may reduce hospital admissions, without affecting mortality rate in CRTd failing heart patients with diabetes. Furthermore, patients in the GLP-1 RA group vs. controls experienced an increase in the CRTd responders rate. In fact, the Kaplan curve shows this effect at 12 months of up in GLP-1 RA group vs. controls, by a significant opening of the scissor at follow up end ( $p$ value $<0.05$ ) (Fig. 4). The entity of CRTd response is based on clinical measures, as patients symptoms, and functional NYHA class assessment, and by echocardiographic diagnosis of LV reverse remodeling [2]. However, GLP-1 RA therapy in addition to standard hypoglycemic drugs, without affecting mortality may control the glycemic homeostasis in CRTd failing heart patients with diabetes, and by other pleiotropic effects may influence ionic channels properties and functions. This may result in the improvement of the electrical stability 


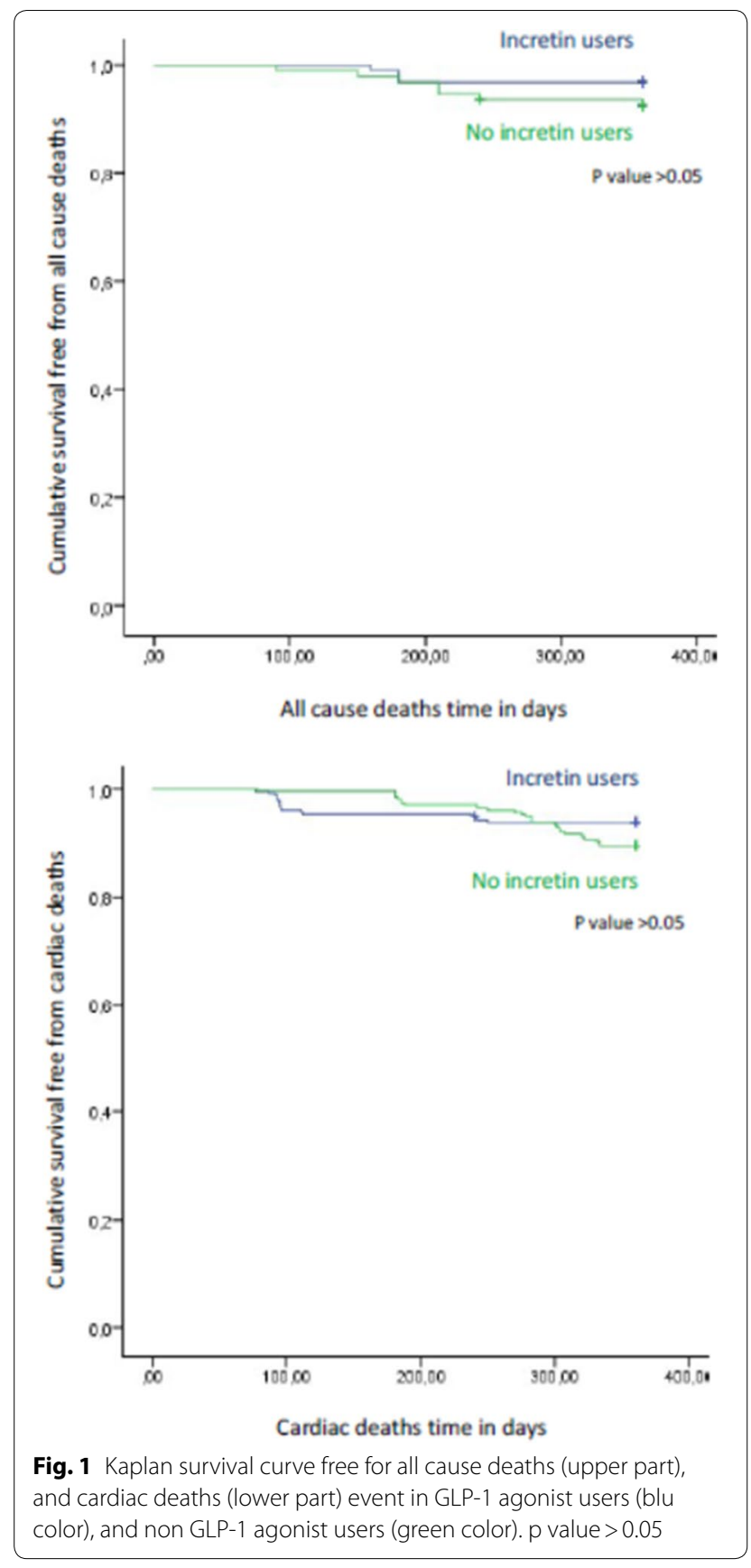

of cardiac membrane, and this may favor the genesis and propagation of cardiac potentials though the cardiac cells. All these effects may improve cardiac electrical, and hemodynamic functions also in patients with severe pump failure due to irreversible cardiac fibrosis. At clinical level, this may be translated in the amelioration of cardiovascular hemodynamic, associated to the reduction of the arrhythmic burden, HF symptoms, and NYHA class. Therefore, GLP-1 RA therapy in addition
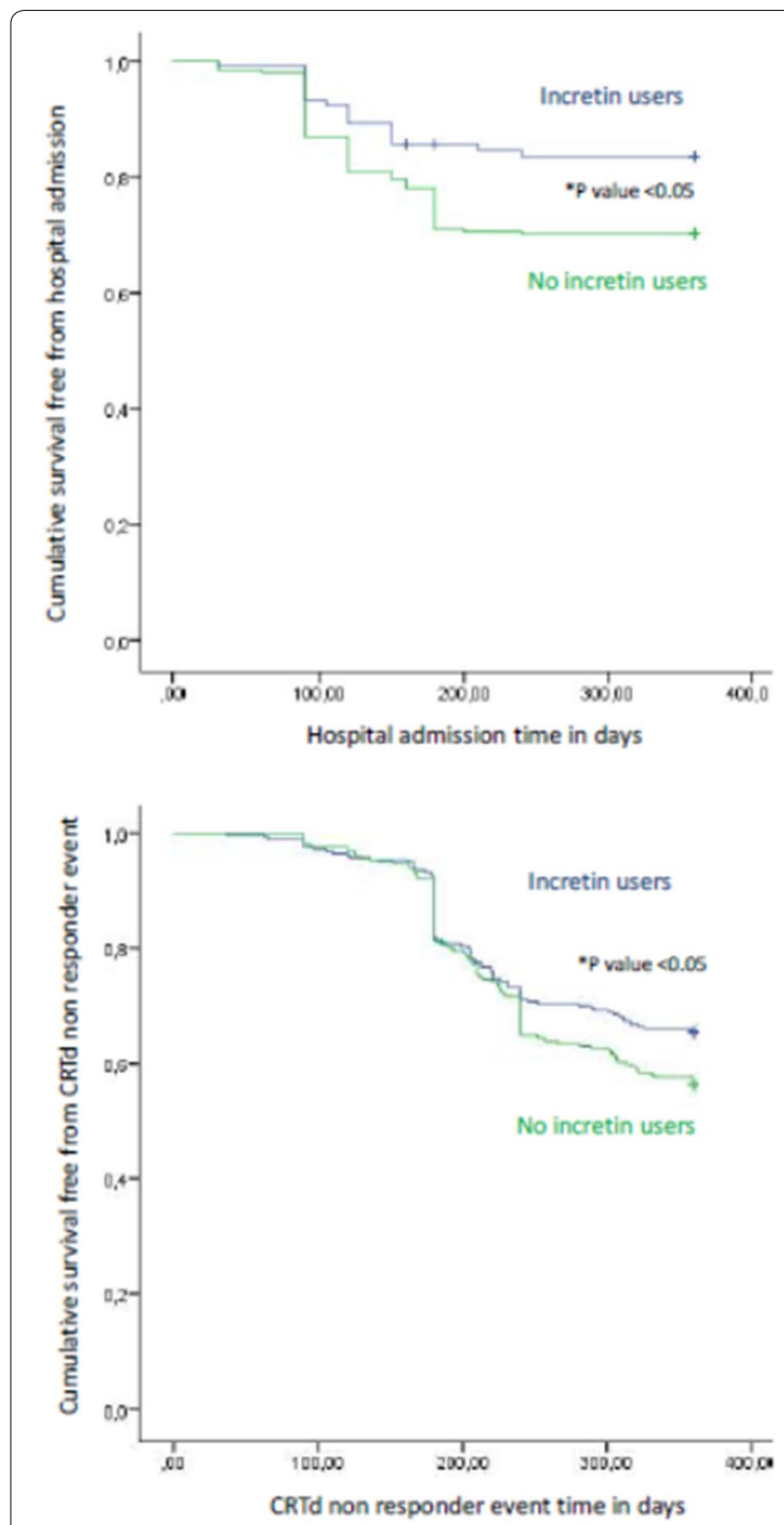

Fig. 2 Kaplan survival curve free for hospital admission (upper part), and cardiac resynchronization with a defibrillator (CRTd) response (lower part) event in GLP-1 agonist users (blu color), and non GLP-1 agonist users (green color). ${ }^{*} p$ value $<0.05$ for both images

to standard hypoglycemic drugs may increase and enhance CRTd response, as a valuable and unique therapeutic effect vs. the conventional hypoglycemic drug treatments.

\section{Study limitations}

All these study result have to be applied in a future study including a larger size of patients with diabetes, and at more long term follow up analysis. In fact, the small 


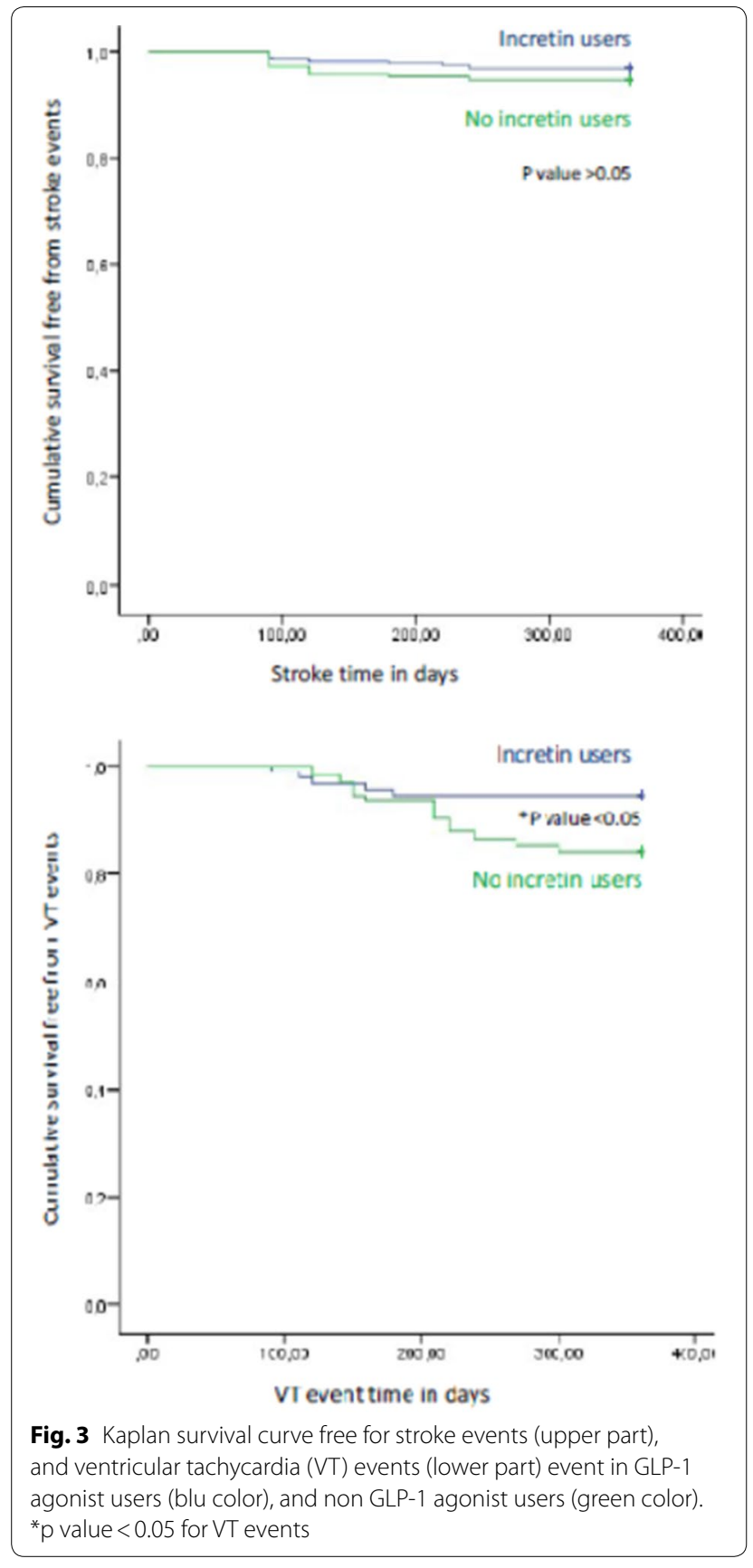

sample size and short duration of follow up may affect study outcomes. In addition, the present study shows data that are not supported by molecular experiments to assess the ionic and molecular alterations GLP-1 RA induced. To date, an animal model of heart failure under hyperglycemic stress condition may lead to the characterization of all these altered cardiac electrical and mechanical processes. In addition, we did not use a continuous monitoring systems for arrhythmias detection

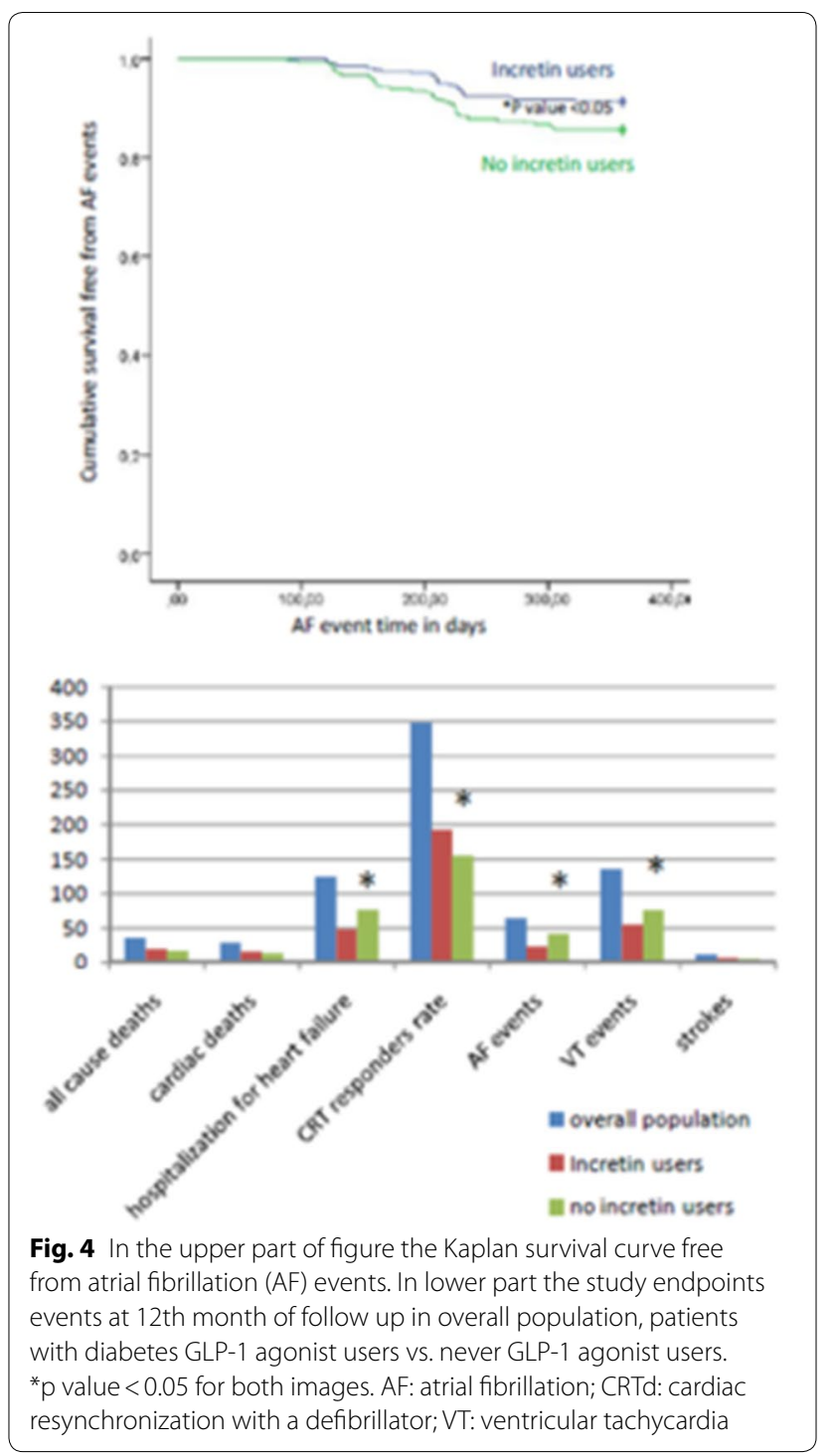

and devices interventions as described by authors [41], and this may affect the study outcomes. We may speculate that, all these pleiotropic and favorable electrical and hemodynamic effects GLP-1 RA induced may be translated in the future treatment of HF in CRTd patients with diabetes. However, further studies are needed to better understand the pleiotropic functions of GLP-1 RA therapy, and their cardiovascular effects. In future, a larger clinical trial may be adequate to assess all these pathogenic processes in a population of failing heart patients with diabetes treated by CRTd. This may be applied in clinical practice to reduce arrhythmic burden, hospitalizations, and to improve CRTd response in failing heart patients with diabetes. 


\section{Table 4 Cox regression analysis for study endpoints}

\begin{tabular}{|c|c|c|c|c|}
\hline & \multicolumn{2}{|l|}{ Univariate analysis } & \multicolumn{2}{|l|}{ Multivariate analysis } \\
\hline & $\mathrm{HR}(95 \% \mathrm{Cl})$ & p value & $\mathrm{HR}(95 \% \mathrm{Cl})$ & p value \\
\hline \multicolumn{5}{|c|}{ a. Multivariate cox regression analysis for parameters associated with all cause deaths } \\
\hline LVEF & $1.060[0.978-1.148]$ & 0.157 & $0.994[0.608-1.625]$ & 0.981 \\
\hline 6MWT & $0.989[0.974-1.003]$ & 0.131 & $0.975[0.869-1.093]$ & 0.661 \\
\hline BNP & $1.001[0.999-1.020]$ & 0.233 & $0.989[0.963-1.016]$ & 0.463 \\
\hline TNF alpha & $1.666[0.650-4.273]$ & 0.288 & $2.253[0.001-2.671]$ & 0.939 \\
\hline GLP-1 & 2.980 [0.904-9.824] & 0.073 & $5.127[0.002-12.548]$ & 0.445 \\
\hline CRP & 0.977 [0.933-1.024] & 0.336 & $0.867[0.002-12.548]$ & 0.548 \\
\hline IL-6 & 1.254 [0.811-1.939] & 0.308 & $0.004[0.545-1.380]$ & 0.961 \\
\hline GLP-1 agonist & $2.373[1.087-5.182]$ & 0.030 & $7.619[0.004-14.031]$ & 0.597 \\
\hline Age & $1.140[1.082-1.201]$ & 0.001 & $1.063[0.805-1.404]$ & 0.665 \\
\hline Obesity & $0.045[0.001-2.916]$ & 0.347 & 1.139 [0.001-3.434] & 0.945 \\
\hline NYHA 3 & $1.518[0.731-3.151]$ & 0.263 & $0.037[0.001-1.358]$ & 0.273 \\
\hline COPD & 3.793 [1.827-7.874] & 0.001 & $0.107[0.001-1.297]$ & 0.529 \\
\hline QRS duration & 1.005 [0.967-1.044] & 0.810 & $0.981[0.608-1.128]$ & 0.081 \\
\hline Hypertension & $1.686[0.689-4.125]$ & 0.252 & $0.005[0.001-2.492]$ & 0.087 \\
\hline Dyslipidemia & $0.957[0.391-2.341]$ & 0.923 & $0.872[0.401-4.951]$ & 0.331 \\
\hline Renal dysfunction & $1.054[0.320-3.476]$ & 0.931 & $3.854[0.015-8.201]$ & 0.095 \\
\hline $\mathrm{Hb} 1 \mathrm{Ac}$ & $0.756[0.435-1.347]$ & 0.354 & $0.416[0.072-2.415]$ & 0.328 \\
\hline \multicolumn{5}{|c|}{ b. Multivariate cox regression analysis for parameters associated with cardiac deaths } \\
\hline LVEF & $0.952[0.902-1.006]$ & 0.080 & $0.438[0.001-8.345]$ & 0.831 \\
\hline 6MWT & $0.998[0.987-1.009]$ & 0.733 & $1.141[0.778-1.673]$ & 0.510 \\
\hline BNP & $1.001[0.989-1.010]$ & 0.853 & $0.993[0.935-1.054]$ & 0.831 \\
\hline TNF alpha & $2.277[1.091-4.752]$ & 0.028 & $0.201[0.011-8.955]$ & 0.727 \\
\hline GLP-1 & $2.980[0.904-9.824]$ & 0.073 & $5.127[0.002-12.548]$ & 0.445 \\
\hline CRP & $0.961[0.920-1.004]$ & 0.075 & $4.282[0.035-5.287]$ & 0.554 \\
\hline IL-6 & 1.364 [0.976-1.906] & 0.069 & $7.623[0.002-12.782]$ & 0.724 \\
\hline GLP-1 agonist & 1.691 [0.929-3.087] & 0.085 & $0.001[0.015-1.491]$ & 0.687 \\
\hline Age & 1.307 [1.237-1.381] & 0.001 & $2.680[0.562-12.791]$ & 0.095 \\
\hline Obesity & 1.018 [0.316-3.282] & 0.976 & $0.013[0.005-6.410]$ & 0.772 \\
\hline NYHA 3 & $8.791[3.474-22.248]$ & 0.001 & $0.010[0.001-7.279$ & 0.633 \\
\hline COPD & $2.041[1.143-3.646]$ & 0.016 & $0.421[0.001-1.760]$ & 0.863 \\
\hline QRS duration & 0.990 [0.959-1.021] & 0.528 & $2.797[0.333-23.510]$ & 0.344 \\
\hline Hypertension & $1.744[0.842-3.614]$ & 0.135 & $0.031[0.001-2.310]$ & 0.868 \\
\hline Dyslipidemia & 3.473 [1.078-11.195] & 0.037 & $4.939[0.001-12.181]$ & 0.358 \\
\hline Renal dysfunction & $0.636[0.197-2.051]$ & 0.449 & 0.052 [0.003-1.193] & 0.422 \\
\hline $\mathrm{Hb} 1 \mathrm{Ac}$ & $1.429[0.911-2.241]$ & 0.120 & 3.119 [0.384-4.091] & 0.167 \\
\hline \multicolumn{5}{|c|}{ c. Multivariate cox regression analysis for parameters associated with hospitalization for heart failure } \\
\hline LVEF & $0.978[0.945-1.012]$ & 0.199 & $0.927[0.826-1.041]$ & 0.202 \\
\hline 6MWT & $1.003[0.997-1.010]$ & 0.301 & $1.013[0.992-1.034]$ & 0.226 \\
\hline BNP & $1.002[1.001-1.020]$ & 0.001 & $1.120[1.001-1.401]$ & $0.017^{*}$ \\
\hline TNF alpha & $1.078[0.665-1.749]$ & 0.761 & $0.028[0.000-1.673]$ & 0.420 \\
\hline GLP-1 & $1.719[1.077-2.744]$ & 0.023 & $1.143[0.451-2.900]$ & 0.778 \\
\hline CRP & 0.987 [0.967-1.007] & 0.207 & $0.981[0.923-1.042]$ & 0.530 \\
\hline IL-6 & $1.163[0.926-1.460]$ & 0.193 & $1.879[0.017-2.129]$ & 0.272 \\
\hline GLP-1 agonist & 1.914 [1.335-2.744] & 0.001 & $0.119[0.028-0.508]$ & $0.004^{*}$ \\
\hline Age & 1.005 [0.979-1.033] & 0.691 & $0.977[0.891-1.072]$ & 0.624 \\
\hline Obesity & 0.997 [0.488-2.039] & 0.994 & $0.923[0.826-1.742]$ & 0.983 \\
\hline NYHA 3 & 1.680 [1.177-2.397] & 0.004 & $1.43[0.398-1.041]$ & 0.562 \\
\hline
\end{tabular}


Table 4 (continued)

\begin{tabular}{|c|c|c|c|c|}
\hline & \multicolumn{2}{|l|}{ Univariate analysis } & \multicolumn{2}{|l|}{ Multivariate analysis } \\
\hline & $\mathrm{HR}(95 \% \mathrm{Cl})$ & $p$ value & $\mathrm{HR}(95 \% \mathrm{Cl})$ & p value \\
\hline COPD & 1.126 [0.776-1.633] & 0.533 & 6.870 [0.639-7.383] & 0.112 \\
\hline QRS duration & $1.005[0.987-1.024]$ & 0.578 & $1.031[0.963-1.102]$ & 0.383 \\
\hline Hypertension & $0.875[0.606-1.265]$ & 0.478 & 1.381 [0.342-5.583] & 0.651 \\
\hline Dyslipidemia & $1.932[1.128-3.311]$ & 0.016 & $0.354[0.081-1.541]$ & 0.166 \\
\hline Renal dysfunction & 0.889 [0.479-1.649] & 0.709 & $0.565[0.103-3.098]$ & 0.510 \\
\hline $\mathrm{Hb} 1 \mathrm{Ac}$ & 1.255 [0.955-1.649] & 0.103 & $0.967[0.626-1.493]$ & 0.879 \\
\hline \multicolumn{5}{|c|}{ d. Multivariate cox regression analysis for parameters associated with CRT responders } \\
\hline LVEF & $1.043[1.019-1.068]$ & 0.001 & $1.032[0.979-1.089]$ & 0.239 \\
\hline 6MWT & $1.010[0.996-1.410]$ & 0.897 & $0.998[0.987-1.008]$ & 0.665 \\
\hline BNP & $1.001[0.989-1.001]$ & 0.487 & $1.012[0.989-1.189]$ & 0.962 \\
\hline TNF alpha & $0.950[0.688-1.312]$ & 0.755 & $0.508[0.007-3.712]$ & 0.757 \\
\hline GLP-1 & $0.840[0.660-1.069]$ & 0.156 & $0.757[0.510-1.123]$ & 0.166 \\
\hline CRP & 0.997 [0.986-1.008] & 0.601 & 0.997 [0.966-1.029] & 0.851 \\
\hline IL-6 & 1.007 [0.857-1.183] & 0.930 & $0.823[0.010-6.662]$ & 0.953 \\
\hline GLP-1 agonist & $1.041[0.841-1.288]$ & 0.714 & $3.707[1.226-14.570]$ & $0.026^{*}$ \\
\hline Age & $0.988[0.972-1.005]$ & 0.154 & 1.010 [0.957-1.045] & 0.992 \\
\hline Obesity & 1.394 [0.934-2.080] & 0.104 & $0.564[0.192-1.656]$ & 0.297 \\
\hline NYHA 3 & $0.711[0.573-0.881]$ & 0.020 & $1.222[0.646-2.313]$ & 0.538 \\
\hline COPD & $0.796[0.624-1.015]$ & 0.066 & $2.474[0.965-6.343]$ & 0.059 \\
\hline QRS duration & 0.989 [0.978-1.001] & 0.053 & $0.999[0.971-1.027]$ & 0.059 \\
\hline Hypertension & $1.073[0.850-1.355]$ & 0.554 & $0.774[0.430-1.394]$ & 0.394 \\
\hline Dyslipidemia & $0.848[0.653-1.102]$ & 0.218 & 1.135 [0.632-2.039] & 0.671 \\
\hline Renal dysfunction & 0.937 [0.651-1.349] & 0.727 & $1.685[0.666-4.260]$ & 0.270 \\
\hline $\mathrm{Hb} 1 \mathrm{Ac}$ & 1.028 [0.864-1.222] & 0.756 & 1.170 [0.949-1.442] & 0.141 \\
\hline \multicolumn{5}{|c|}{ e. Multivariate cox regression analysis for parameters associated with strokes } \\
\hline LVEF & $0.931[0.866-1.002]$ & 0.055 & $0.449[0.033-7.319]$ & 0.758 \\
\hline 6MWT & $0.989[0.973-1.006]$ & 0.203 & $1.065[0.232-4.894]$ & 0.935 \\
\hline BNP & $1.001[0.989-1.020]$ & 0.297 & $0.991[0.809-1.214]$ & 0.932 \\
\hline TNF alpha & $1.062[0.347-3.250]$ & 0.917 & $0.016[0.001-6.694]$ & 0.988 \\
\hline GLP-1 & $2.315[0.69-7.761]$ & 0.174 & $0.020[0.001-7.072]$ & 0.862 \\
\hline CRP & $0.983[0.935-1.033]$ & 0.502 & $1.215[0.002-7.735]$ & 0.953 \\
\hline IL-6 & 0.617 [0.273-1.394] & 0.245 & $2.159[0.001-3.829]$ & 0.991 \\
\hline GLP-1 agonist & $1.691[0.740-3.864]$ & 0.213 & $1.320[0.001-6.473]$ & 0.913 \\
\hline Age & $0.946[0.885-1.012]$ & 0.104 & $1.263[0.004-4.136]$ & 0.937 \\
\hline Obesity & 5.241 [2.080-13.205] & 0.001 & $0.031[0.001-2.678]$ & 0.771 \\
\hline NYHA 3 & $0.994[0.447-2.213]$ & 0.989 & $0.129[0.001-5.426]$ & 0.960 \\
\hline COPD & 1.764 [0.784-3.972] & 0.170 & $0.212[0.001-1.878]$ & 0.896 \\
\hline QRS duration & 1.001 [0.960-1.045] & 0.951 & 1.315 [0.059-2.940] & 0.960 \\
\hline Hypertension & $1.264[0.502-3.185]$ & 0.619 & $2.066[0.001-11.954]$ & 0.982 \\
\hline Dyslipidemia & $0.699[0.277-1.762]$ & 0.448 & $0.948[0.001-3.020]$ & 0.794 \\
\hline Renal dysfunction & $0.043[0.001-16.543]$ & 0.301 & 0.880 [0.001-1.003] & 0.880 \\
\hline $\mathrm{Hb} 1 \mathrm{Ac}$ & $1.115[0.412-3.021]$ & 0.830 & $4.248[0.001-5.346]$ & 0.752 \\
\hline \multicolumn{5}{|c|}{ f. Multivariate cox regression analysis for parameters associated with VT events } \\
\hline LVEF & $1.041[0.985-1.100]$ & 0.157 & $1.160[1.012-1.290]$ & $0.047^{*}$ \\
\hline 6MWT & $0.993[0.983-1.040]$ & 0.203 & $0.993[0.983-1.004]$ & 0.194 \\
\hline BNP & $1.001[0.909-1.020]$ & 0.321 & $1.001[0.098-1.002]$ & 0.505 \\
\hline TNF alpha & $1.475[0.749-2.904]$ & 0.261 & $1.009[0.484-2.101]$ & 0.981 \\
\hline GLP-1 & $1.839[0.904-3.739]$ & 0.093 & $0.878[0.585-1.317]$ & 0.529 \\
\hline
\end{tabular}


Table 4 (continued)

\begin{tabular}{|c|c|c|c|c|}
\hline & \multicolumn{2}{|l|}{ Univariate analysis } & \multicolumn{2}{|c|}{ Multivariate analysis } \\
\hline & $\mathrm{HR}(95 \% \mathrm{CI})$ & $p$ value & HR $(95 \% \mathrm{Cl})$ & $p$ value \\
\hline CRP & 0.973 [0.940-1.007] & 0.121 & 0.991 [0.958-1.025] & 0.609 \\
\hline IL-6 & 1.384 [1.034-1.853] & 0.029 & $1.076[0.780-1.485]$ & 0.654 \\
\hline GLP-1 agonist & $0.332[0.185-0.597]$ & 0.001 & $0.964[0.963-0.992]$ & $0.012^{*}$ \\
\hline Age & $1.039[1.001-1.079]$ & 0.046 & 1.051 [0.816-1.599] & 0.066 \\
\hline Obesity & 5.478 [3.005-9.987] & 0.001 & 0.174 [0.016-1.599] & 0.072 \\
\hline NYHA 3 & 2.773 [1.562-4.923] & 0.001 & $0.541[0.281-1.043]$ & 0.067 \\
\hline COPD & 2.345 [1.404-3.915] & 0.001 & $0.846[0.466-1.538]$ & 0.584 \\
\hline QRS duration & $0.975[0.948-1.003]$ & 0.080 & $1.511[1.160-1.959]$ & $0.026^{*}$ \\
\hline Hypertension & $0.805[0.469-1.380]$ & 0.430 & $1.031[0.570-1.863]$ & 0.920 \\
\hline Dyslipidemia & 2.619 [1.048-6.548] & 0.039 & $1.232[0.715-2.122]$ & 0.452 \\
\hline Renal dysfunction & $1.056[0.454-2.456]$ & 0.901 & $0.420[0.012-1.531]$ & 0.075 \\
\hline $\mathrm{Hb} 1 \mathrm{Ac}$ & 1.239 [0.760-2.020] & 0.389 & $1.320[0.021-3.426]$ & 0.752 \\
\hline \multicolumn{5}{|c|}{ g. Multivariate cox regression analysis for parameters associated with AF events } \\
\hline LVEF & $0.964[0.934-0.994]$ & 0.021 & $1.593[1.122-1.986]$ & $0.006^{*}$ \\
\hline 6MWT & $1.001[0.994-1.601]$ & 0.977 & $1.024[0.995-1.046]$ & 0.712 \\
\hline BNP & $1.020[1.001-1.100]$ & 0.008 & $1.101[1.001-1.210]$ & $0.017^{*}$ \\
\hline TNF alpha & $1.180[0.759-1.836]$ & 0.462 & $1.298[0.773-2.180]$ & 0.324 \\
\hline GLP-1 & $1.049[0.720-1.526]$ & 0.804 & $1.087[0.829-1.427]$ & 0.962 \\
\hline CRP & $1.010[0.983-1.180]$ & 0.561 & $0.997[0.979-1.015]$ & 0.732 \\
\hline IL-6 & 1.016 [0.808-1.277] & 0.983 & $0.936[0.703-1.247]$ & 0.652 \\
\hline GLP-1 agonist & $0.577[0.348-0.957]$ & 0.033 & $0.603[0.411-0.884]$ & $0.010^{*}$ \\
\hline Age & 0.989 [0.964-1.014] & 0.388 & $0.982[0.957-1.008]$ & 0.182 \\
\hline Obesity & $0.941[0.479-1.846]$ & 0.859 & $0.952[0.493-1.841]$ & 0.885 \\
\hline NYHA 3 & $1.044[0.756-1.441]$ & 0.795 & $0.942[0.670-1.324]$ & 0.730 \\
\hline COPD & 1.113 [0.785-1.577] & 0.548 & $1.020[0.683-1.523]$ & 0.924 \\
\hline QRS duration & 1.020 [1.003-1.037] & 0.020 & $1.182[1.012-1.552]$ & $0.043^{*}$ \\
\hline Hypertension & $0.929[0.656-1.316]$ & 0.680 & 1.519 [0.926-2.492] & 0.098 \\
\hline Dyslipidemia & $0.828[0.560-1.223]$ & 0.343 & $1.232[0.715-2.122]$ & 0.452 \\
\hline Renal dysfunction & 0.588 [0.598-1.162] & 0.126 & $0.613[0.024-1.618]$ & 0.082 \\
\hline $\mathrm{Hb} 1 \mathrm{Ac}$ & $1.176[0.914-1.512]$ & 0.208 & $1.150[0.002-2.152]$ & 0.073 \\
\hline
\end{tabular}

GLP-1: glucagone like peptide 1; COPD: chronic obstructive pulmonary disease; NYHA: New York Heart Association; 6MWT: 6 min walking test; CRTd: cardiac resynchronization with a defibrillator; $n$.s.: not statistical significant; LVEF: left ventricle ejection fraction; ACE: angiotensin converting enzyme; ARS: angiotensin receptor; NOAC: new oral anticoagulant; DPP4: Di-Peptidil-Peptidasi IV; BNP: B type natriuretic peptide; CRP: C reactive protein; IL6: interleukine 6; TNFa: tumor necrosis factor alpha

$p$ value $<0.05$ is statistical significant, and marked as *

\section{Conclusions}

GLP-1 RA therapy in addition to standard hypoglycemic drugs vs. standard hypoglycemic drugs may significantly reduce inflammation, and BNP values in failing heart patients with diabetes treated by CRTd. These antiinflammatory and hemodynamics effects are linked to significant improvement of LVEF, and to the reduction of the NYHA class, arrhythmic burden, and hospitalization for HF worsening. Intriguingly, GLP-1 RA therapy in addition to standard hypoglycemic drugs is associated to a 3.7 fold higher rate of CRTd responders vs. other conventional hypoglycemic drugs. Therefore, GLP-1
RA therapy in addition to standard hypoglycemic drugs may improve CRT responder rate and clinical outcomes in patients with diabetes. However, GLP-1 RA therapy in addition to standard hypoglycemic drugs may be recommended in T2DM failing heart patients treated by CRT-d.

\footnotetext{
Abbreviations

AF: atrial fibrillation; BNP: B type natriuretic peptide; CRP: C reactive protein; CRT-d: cardiac resynchronization therapy with a defibrillator; DPP-4: dipeptidyl peptidase-4; ELISA: enzyme-linked immunosorbent assay; GLP-1: glucagonlike peptide 1; GLP-1 RA: glucagon-like peptide 1 receptor agonists; HF: heart failure; HFrEF: heart failure and reduced left ventricle ejection fraction; ICD:
} 
internal cardioverter defibrillator; IL6: interleukine 6; LV: left ventricle; LVEF: left ventricle ejection fraction; NYHA: New York Association Heart;T2DM: type 2 diabetes mellitus; TNFa: tumor necrosis factor alpha; VF: ventricular fibrillation; VT: ventricular tachycardia.

\section{Authors' contributions}

CS: wrote the research project and the full manuscript. PP: data collection and interpretation; CS, MS, AR, and CM: performer CRTd implants; CDL: study revision; GP, MRR, MB: study revision editing, and data analysis; RM: manuscript editing. All authors read and approved the final manuscript.

\section{Author details \\ ${ }^{1}$ Department of Medical, Surgical, Neurological, Metabolic and Aging Sci- ences, University of Campania "Luigi Vanvitelli", Piazza Miraglia, 2, 80138 Naples, Italy. ${ }^{2}$ Cardiovascular and Arrhythmias Department, John Paul II Research and Care Foundation, Campobasso, Italy. ${ }^{3}$ Center for Translational Medicine, Temple University, Philadelphia, USA. ${ }^{4}$ Cardiovascular Diseases Department, Cardarelli Hospital, Naples, Italy.}

\section{Acknowledgements}

All authors have equally contributed to this research. This research did not receive any specific grant from funding agencies in the public, commercial, or not-for-profit sectors.

\section{Competing interests}

C.S edited and wrote the research project and the full manuscript.

\section{Availability of data and materials}

Data and study materials are available.

\section{Consent for publication}

Authors give the full consent to publish the present article.

\section{Ethics approval and consent to participate}

Ethical Committee of University of Campania "Luigi Vanvitelli", Catholic University of Sacred Heart, John Paul II Research and Care Foundation, and Cardarelli Hospital approved the research protocol and gave the consent to participate in the study.

\section{Funding}

None to declare.

\section{Publisher's Note}

Springer Nature remains neutral with regard to jurisdictional claims in published maps and institutional affiliations.

Received: 10 August 2018 Accepted: 10 October 2018

Published online: 22 October 2018

\section{References}

1. Lehrke M, Marx N. Diabetes mellitus and heart failure. Am J Cardiol. 2017;120(1S):S37-47.

2. Yancy CW, Jessup M, Bozkurt B, Butler J, Casey DE Jr, Colvin MM, Drazner MH, Filippatos GS, Fonarow GC, Givertz MM, Hollenberg SM, Lindenfeld J, Masoudi FA, McBride PE, Peterson PN, Stevenson LW, Westlake C. 2017 ACC/AHA/HFSA focused update of the 2013 ACCF/AHA guideline for the management of heart failure: a report of the American College of Cardiology/American Heart Association Task Force on Clinical Practice Guidelines and the Heart Failure Society of America. J Am Coll Cardiol. 2017;70(6):776-803.

3. MacDonald MR, Petrie MC, Varyani F, Ostergren J, Michelson EL, Young JB, Solomon SD, Granger CB, Swedberg K, Yusuf S, Pfeffer MA, McMurray $\mathrm{JJ}$, CHARM Investigators. Impact of diabetes on outcomes in patients with low and preserved ejection fraction heart failure: an analysis of the Candesartan in Heart failure: assessment of reduction in mortality and morbidity (CHARM) programme. Eur Heart J. 2008;29(11):1377-85.

4. Sardu C, Barbieri M, Santamaria M, Giordano V, Sacra C, Paolisso P, Spirito A, Marfella R, Paolisso G, Rizzo MR. Multipolar pacing by cardiac resynchronization therapy with a defibrillators treatment in type 2 diabetes mellitus failing heart patients: impact on responders rate, and clinical outcomes. Cardiovasc Diabetol. 2017;16(1):75.

5. Filion KB, Azoulay L, Platt RW, Dahl M, Dormuth CR, Clemens KK, Hu N, Paterson JM, Targownik L, Turin TC, Udell JA, Ernst P, for the CNODES Investigators. A multicenter observational study of GLP-1 agonist-based drugs and heart failure. N Engl J Med. 2016;374:1145-54.

6. Moghissi ES, Korytkowski MT, DiNardo M, Einhorn D, Hellman R, Hirsch IB, Inzucchi SE, Ismail-Beigi F, Kirkman MS, Umpierrez GE, American Association of Clinical Endocrinologists, American Diabetes Association. American Association of Clinical Endocrinologists and American Diabetes Association consensus statement on inpatient glycemic control. Diabetes Care. 2009;32(6):1119-31.

7. Marfella R, Sardu C, Balestrieri ML, Siniscalchi M, Minicucci F, Signoriello G, Calabrò P, Mauro C, Pieretti G, Coppola A, Nicoletti G, Rizzo MR, Paolisso G, Barbieri M. Effects of GLP-1 agonist treatment on cardiovascular outcomes in diabetic STEMI-patients with culprit obstructive and multivessel non obstructive-coronary-stenosis. Diabetol Metab Syndr. 2018;10:1.

8. Marfella R, Sardu C, Calabrò P, Siniscalchi M, Minicucci F, Signoriello G, Balestrieri ML, Mauro C, Rizzo MR, Paolisso G, Barbieri M. Non-ST-elevation myocardial infarction outcomes in patients with type 2 diabetes with non-obstructive coronary artery stenosis: effects of GLP-1 agonist treatment. Diabetes Obes Metab. 2018;20(3):723-9.

9. Sardu C, Santamaria M, Funaro S, Sacra C, Barbieri M, Paolisso P, Marfella R, Paolisso G, Rizzo MR. Cardiac electrophysiological alterations and clinical response in cardiac resynchronization therapy with a defibrillator treated patients affected by metabolic syndrome. Medicine (Baltimore). 2017;96(14):e6558

10. Petretta M, Colao A, Sardu C, Scopacasa F, Marzullo P, Pivonello R, Fontanella L, de Caterina M, de Simone A, Bonaduce D. NT-proBNP, IGF-I and survival in patients with chronic heart failure. Growth Horm IGF Res. 2007;17(4):288-96.

11. Anand IS, Fisher LD, Chiang YT, et al. Changes in brain natriuretic peptide and norepinephrine over time and mortality and morbidity in the valsartan heart failure trial (Val-HeFT). Circulation. 2003;107:1278-83.

12. Moertl D, Berger R, Struck J, et al. Comparison of midregional pro-atrial and B-type natriuretic peptides in chronic HF: influencing factors, detection of left ventricular systolic dysfunction, and prediction of death. J Am Coll Cardiol. 2009:53:1783-90.

13. Tolosana JM, Madrid AH, Brugada J, Sitges M, Bolao IG, Lozano IF, Ferrer JM, Quesada A, Macias A, Marin W, Escudier JM, Gomez AA, Gimenez AM, Tamborero D, Berruezo A, Mont L. Comparison of benefits and mortality in cardiac resynchronization therapy in patients with atrial fibrillation versus patients in sinus rhythm (Results of the Spanish Atrial Fibrillation and Resynchronization [SPARE] Study). Am J Cardiol. 2008;102(4):444-9.

14. Tuinenburg AE, Brundel BJ, Van Gelder IC, Henning RH, Van Den Berg MP, Driessen C, Grandjean JG, Van Gilst WH, Crijns HJ. Gene expression of the natriuretic peptide system in atrial tissue of patients with paroxysmal and persistent atrial fibrillation. J Cardiovasc Electrophysiol. 1999;10:827-35.

15. Patton KK, Ellinor PT, Heckbert SR, Christenson RH, DeFilippi C, Gottdiener $J S$, Kronmal RA. N-terminal pro-B-type natriuretic peptide is a major predictor of the development of atrial fibrillation: the Cardiovascular Health Study. Circulation. 2009;120:1768-74.

16. Marfella R, Di Filippo C, Potenza N, Sardu C, Rizzo MR, Siniscalchi M, Musacchio E, Barbieri M, Mauro C, Mosca N, Solimene F, Mottola MT, Russo A, Rossi F, Paolisso G, D'Amico M. Circulating microRNA changes in heart failure patients treated with cardiac resynchronization therapy: responders vs. non-responders. Eur J Heart Fail. 2013;15(11):1277-88.

17. Sardu C, Marfella R, Santulli G, Paolisso G. Functional role of miRNA in cardiac resynchronization therapy. Pharmacogenomics. 2014;15(8):1 159-68.

18. Sardu C, Barbieri M, Rizzo MR, Paolisso P, Paolisso G, Marfella R. Cardiac resynchronization therapy outcomes in type 2 diabetic patients: role of microRNA changes. J Diabetes Res. 2016;2016:7292564.

19. Sardu C, Marfella R, Santulli G. Impact of diabetes mellitus on the clinical response to cardiac resynchronization therapy in elderly people. J Cardiovasc Transl Res. 2014;7(3):362-8.

20. Cikes M, Solomon SD. Beyond ejection fraction: an integrative approach for assessment of cardiac structure and function in heart failure. Eur Heart J. 2016;37(21):1642-50. 
21. Schliamser JE, Kadish AH, Subacius H, et al. Significance of follow-up left ventricular ejection fraction measurements in the Defibrillators in NonIschemic Cardiomyopathy Treatment Evaluation trial (DEFINITE). Heart Rhythm. 2013;10:838-46.

22. Kini $V$, Soufi MK, Deo R, et al. Appropriateness of primary prevention implantable cardioverter defibrillators at the time of generator replacement: are indications still met? J Am Coll Cardiol. 2014;63:2388-94.

23. Cheng A, Dalal D, Butcher B, et al. Prospective observational study of implantable cardioverter defibrillators in primary prevention of sudden cardiac death: study design and cohort description. J Am Heart Assoc. 2013;2:e000083.

24. Ghio S, Constantin C, Klersy C, et al. Interventricular and intraventricular dyssynchrony are common in heart failure patients, regardless of QRS duration. Eur Heart J. 2004;25(7):571-8.

25. Young JB, Abraham WT, Smith AL, et al. Combined cardiac resynchronization and implantable cardioversion defibrillation in advanced chronic heart failure: the MIRACLE ICD Trial. JAMA. 2003;289(20):2685-94.

26. Bax JJ, Abraham T, Barold SS, et al. Cardiac resynchronization therapy: part 1-issues before device implantation. J Am Coll Cardiol. 2005;46(12):2153-67.

27. Higgins SL, Hummel JD, Niazi IK, et al. Cardiac resynchronization therapy for the treatment of heart failure in patients with intraventricular conduction delay and malignant ventricular tachyarrhythmias. J Am Coll Cardiol. 2003;42(8):1454-9.

28. Bristow MR, Feldman AM, Saxon LA. Heart failure management using implantable devices for ventricular resynchronization: comparison of medical therapy, pacing, and defibrillation in chronic heart failure (COMPANION) trial. COMPANION Steering Committee and COMPANION Clinical Investigators. J Card Fail. 2000;6(3):276-85.

29. Auricchio A, Stellbrink C, Sack S, et al. The pacing therapies for congestive heart failure (PATH-CHF) study: rationale, design, and endpoints of a prospective randomized multicenter study. Am J Cardiol. 1999:83(5B):130D-5D.

30. Stellbrink C, Auricchio A, Butter $C$, et al. Pacing therapies in congestive heart failure II study. Am J Cardiol. 2000;86(9A):138K-43K.

31. Cleland JG, Daubert JC, Erdmann E, et al. The CARE-HF study (CArdiac REsynchronisation in Heart Failure study): rationale, design and endpoints. Eur J Heart Fail. 2001;3(4):481-9.

32. Abraham WT. Rationale and design of a randomized clinical trial to assess the safety and efficacy of cardiac resynchronization therapy in patients with advanced heart failure: the Multicenter InSync Randomized Clinical Evaluation (MIRACLE). J Card Fail. 2000;6(4):369-80.

33. Linde C, Leclercq C, Rex S, et al. Long-term benefits of biventricular pacing in congestive heart failure: results from the MUltisite STimulation in cardiomyopathy (MUSTIC) study. J Am Coll Cardiol. 2002;40(1):111-8.

34. Leclerca C, Victor F, Alonso C, et al. Comparative effects of permanent biventricular pacing for refractory heart failure in patients with stable sinus rhythm or chronic atrial fibrillation. Am J Cardiol. 2000;85(9):11541156, A9.

35. Szepietowska B, Polonsky B, Sherazi S, et al. Effect of obesity on the effectiveness of cardiac resynchronization to reduce the risk of first and recurrent ventricular tachyarrhythmia events. Cardiovasc Diabetol. 2016;15:93.

36. Marzec LN, Peterson PN, Bao H, et al. Use of cardiac resynchronization therapy among eligible patients receiving an implantable cardioverter defibrillator insights from the National cardiovascular data registry implantable cardioverter defibrillator registry. JAMA Cardiol. 2017;2(5):561-5.

37. Santulli G, Pagano G, Sardu C, Xie W, Reiken S, D'Ascia SL, Cannone M, Marziliano N, Trimarco B, Guise TA, Lacampagne A, Marks AR. Calcium release channel RyR2 regulates insulin release and glucose homeostasis. J Clin Invest. 2015;125(11):4316.

38. Sardu C, Carreras G, Katsanos S, Kamperidis V, Pace MC, Passavanti MB, Fava I, Paolisso P, Pieretti G, Nicoletti GF, Santulli G, Paolisso G, Marfella R. Metabolic syndrome is associated with a poor outcome in patients affected by outflow tract premature ventricular contractions treated by catheter ablation. BMC Cardiovasc Disord. 2014;14:176.

39. Sokos GG, Nikolaidis LA, Mankad S, Elahi D, Shannon RP. Glucagonlike peptide-1 infusion improves left ventricular ejection fraction and functional status in patients with chronic heart failure. J Card Fail. 2006;12(9):694-9.

40. Nathanson D, Ullman B, Löfström U, Hedman A, Frick M, Sjöholm A, et al. Effects of intravenous exenatide in type 2 diabetic patients with congestive heart failure: a double-blind, randomized controlled clinical trial of efficacy and safety. Diabetologia. 2012;55(4):926-35.

41. Sardu C, Santamaria M, Rizzo MR, Barbieri M, di Marino M, Paolisso G, Santulli G, Marfella R. Telemonitoring in heart failure patients treated by cardiac resynchronisation therapy with defibrillator (CRT-D): the TELECART Study. Int J Clin Pract. 2016;70(7):569-76.
Ready to submit your research? Choose BMC and benefit from:

- fast, convenient online submission

- thorough peer review by experienced researchers in your field

- rapid publication on acceptance

- support for research data, including large and complex data types

- gold Open Access which fosters wider collaboration and increased citations

- maximum visibility for your research: over 100M website views per year

At BMC, research is always in progress.

Learn more biomedcentral.com/submissions 